\title{
退火前后镍铇硼合金电沉积层的结构与性能 *
}

\author{
曹刚敏 杨防祖 黄 令 牛振江 许书楷 周绍民
}

(厦门大学化学系 固体表面物理化学国家重点实验室 物理化学研究所, 厦门 361005)

\begin{abstract}
摘要 采用电化学技术、XPS、DSC、XRD 等方法研究 Ni-W-B 合金电沉积及热处理前后合金镀层的结构和显微 硬度. 结果表明, 在 Ni-W-B 合金电沉积过程中伴随着化学沉积镍等过程以及 $\mathrm{Na}_{2} \mathrm{~B}_{4} \mathrm{O}_{7}$ 在镀层中的夹杂; Ni-W 和 Ni-W-B 合金电沉积层分别表现为纳米晶结构和非晶态结构; 热处理过程中合金电沉积层发生晶粒粗化过程 以及 Ni-W-B 合金镀层发生新相形成过程, 产生 $\mathrm{Ni}_{4} \mathrm{~W}$ 和镍嗍化物如 $\mathrm{Ni}_{2} \mathrm{~B} 、 \mathrm{Ni}_{3} \mathrm{~B}$ 等沉淀物; $400{ }^{\circ} \mathrm{C}$ 热处理 $2 \mathrm{~h}$ 后 $\mathrm{Ni}-\mathrm{W}$ 合金镀层有最大的显微硬度达 $919.8 \mathrm{~kg} \cdot \mathrm{mm}^{-2}$, 而在 $500{ }^{\circ} \mathrm{C}$ 下 Ni-W-B 合金有最大的硬度达 $1132.2 \mathrm{~kg} \cdot$ $\mathrm{mm}^{-2}$.
\end{abstract}

关键词: Ni-W-B 合金, 电沉积, 结构, 显微硬度, 热处理

铬镀层由于悦目的表观, 良好的耐磨、耐蚀性能 而被使用了一个多世纪。然而铬镀液不仅有毒，而 且有腐蚀性。因此, 开发代铬镀层已成为迫切需要 解决的问题。近年来, 镍基合金特别是 Ni-W 合金 与 Ni-W-B 合金的研究格外引人注目 .

铇无法从水溶液中单独电沉积, 而可与镍诱导 共沉积. Ni-W 合金镀层 ${ }^{[1-6]}$ 具有优良的耐蚀性、耐 热性和耐磨性，可使用于磨损条件下腐蚀环境中作 为如轴承、活塞、汽缸和石油工业中某些产品的表 面镀层。在 $\mathrm{Ni}-\mathrm{W}$ 合金镀液中若加人含 $\mathrm{B}$ 物质, 可 以得到非晶态 Ni-W-B 合金镀层，比晶态合金有更 优异的耐蚀和耐磨等性能.

有关 Ni-W-B 合金电沉积及镀层结构与性能已 有研究报导 ${ }^{[7-12]}$, Croopnick ${ }^{[11]}$ 等撰文说明, 具有组 成为 $59.5 \% \mathrm{Ni}-39.5 \% \mathrm{~W}-1 \% \mathrm{~B}$ (质量分数, 下同) 的非晶态 Ni-W-B 合金镀层的物理和化学性能相当 于铬镀层, 显微硬度为 $H_{v} 600 \mathrm{~kg} \cdot \mathrm{mm}^{-2}$ 左右, 具 有极佳的韧性和优于 $\mathrm{Cr}$ 的抗腐蚀性能, 经过热处 理后硬度可达 $H_{v} 1000 \mathrm{~kg} \cdot \mathrm{mm}^{-2}$ 左右. Cretchen 等 ${ }^{[10]}$ 研究结果表明, 少量 B 元素的引入增大了合 金的无序程度，并使镀层的显微硬度提高, $\mathrm{B}$ 的引 人不会在镀层中形成新相。含 $\mathrm{W} 31 \%, \mathrm{~B} 1 \%$ 的
Ni-W-B 合金具有亚稳态的纳米晶 - 非晶态结构,经 热处理后, 镀层的晶化程度提高, 晶粒尺寸增大, 产 生新相 $\mathrm{Ni}_{4} \mathrm{~W}$, 镀层的显微硬度从 $H_{v} 600$ 上升到 $H_{v}$ 900 左右.

本文在柠檬酸铵中性 $\mathrm{Ni}-\mathrm{W}$ 合金镀液中加人二 甲基胺硼烷 $\left(\mathrm{C}_{2} \mathrm{H}_{10} \mathrm{BN}\right)$, 探索二甲基胺硼烷在 $\mathrm{Ni}-\mathrm{W}$ 合金电沉积过程中的作用, 分别获得 $\mathrm{Ni}-\mathrm{W}$ 和 Ni-W-B 合金电沉积层, 揭示它们在退火前后的结 构差异和显微硬度的变化。

\section{1 实验方法}

镀液组成 $\left(\mathbf{g} \cdot \mathbf{L}^{-1}\right)$ 和实验条件: $\mathrm{NiSO}_{4}$. $6 \mathrm{H}_{2} \mathrm{O} 30, \mathrm{Na}_{2} \mathrm{WO}_{4} \cdot 2 \mathrm{H}_{2} \mathrm{O} 65,\left(\mathrm{NH}_{4}\right)_{3} \mathrm{C}_{6} \mathrm{H}_{5} \mathrm{O}_{7} 100$, $\mathrm{C}_{2} \mathrm{H}_{10} \mathrm{BN}$ 15. 用化学纯试剂和去离子水配制溶液, 沉积条件: $60{ }^{\circ} \mathrm{C}, \mathrm{pH} 7.0 \sim 7.5$, 阳极为石墨板, 阴 极为紫铜片 $(99.99 \%)$, 工作面积为 $(2.0 \mathrm{~cm} \times 3.0$ $\mathrm{cm}) \times 2$ 双面镀。镀液用磁力加热搅拌器中速摚 拌, 沉积电流密度 $2.0 \mathrm{~A} \cdot \mathrm{dm}^{-2}$, 沉积时间 $150 \mathrm{~min}$.

热处理：将镀片置于上海实验电炉厂 SK2-1-10H 回转式管式电阻炉中在 $\mathrm{Ar}$ 气氛下分别 在 $400{ }^{\circ} \mathrm{C}$ 和 $500{ }^{\circ} \mathrm{C}$ 下热处理 $120 \mathrm{~min}$. 镀层热处理 (DSC) 在德国 NETZSCH DSC204 热分析测试仪上

2000-05-08 收到初稿, 2000-09-05 收到修改稿. 联系人：杨防祖（E-mail: smzhou@ @mu. edu. cn）。*国家自然科学基金资助项目 (29773039) 
进行, $\mathrm{N}_{2}$ 气氛, 升温速率 $20{ }^{\circ} \mathrm{C} \cdot \mathrm{min}^{-1}$.

镀层的组成: 镀层 $\mathrm{Ni}$ 含量采用分光光度法测 定 ${ }^{[13]}$, 镀层剥离, 经浓硝酸溶解和进一步处理后, 用丁二䏡为络合剂，在氨介质中测定波长为 445 $\mathrm{nm}$ 时的吸光度.

镀层 $\mathbf{B}$ 状态和组成: 在 VG 公司 ESCALABMK- II电子能谱（XPS）仪上进行, 真空度优于 $10^{-8} \mathrm{~Pa}$, 以 $\mathrm{Al} K_{\alpha}$ 射线作为激发源，通过能为 100 $\mathrm{eV}, \mathrm{Ar}^{+}$离子射电压为 $11.00 \mathrm{kV}$, 射电流为 $20 \mathrm{~mA}$, 以 $\mathrm{Cl} s$ 结合能 $284.6 \mathrm{eV}$ 为能量校正标准.

镀层的结构: 用日本理学公司 D.MAX-RC 多 晶转靶 $\mathrm{X}$ 射线衍射仪 (XRD) 测定. 采用 $\mathrm{Cu} K_{\alpha}$ 靶, $\lambda$ 为 $0.15406 \mathrm{~nm}$, 管流 $30 \mathrm{~mA}$, 管压 $40 \mathrm{kV}$, 狭缝系 统为 $1^{\circ} \mathrm{DS}-1^{\circ} \mathrm{SS}-0.15 \mathrm{mmRS}$, 以石墨单色器滤波, 扫描速度 $8^{\circ} \cdot \mathrm{min}^{-1}$.

镀层显微硬度:用上海第二光学仪器厂的 71 型 显微硬度计测定. 负荷为 $100 \mathrm{~g}$, 接触时间为 $15 \mathrm{~s}$.

用下列公式计算合金电沉积层的结构参数:

$$
\text { 晶粒尺寸 } D_{h k l}=k \lambda /(\beta \cos \theta)
$$

晶面间距 $d$ 与掠射角 $\theta$ 的关系 $\lambda=2 d \cdot \sin \theta \quad$ (2) 式中 $k$ 为 Scherrer 常数, 取 $1 . \lambda$ 为 $\mathrm{X}$ 射线波长, 对 $\mathrm{Cu}$ 靶 $\lambda=0.15406 \mathrm{~nm} . \theta$ 为 Bragg 角. $\beta$ 为衍射峰 的半峰宽宽化程度 $(\mathrm{rad})$.

合金电沉积的电流效率:

$\eta=\eta_{\mathrm{w}}+\eta_{\mathrm{Ni}}=\frac{w_{\mathrm{w}} \Delta G / E_{\mathrm{W}}}{I t}+\frac{w_{\mathrm{Ni}} \Delta G / E_{\mathrm{Ni}}}{I t}$

式中 $\eta_{\mathrm{W}} 、 \eta_{\mathrm{Ni}}$ 为 $\mathrm{W}$ 和 $\mathrm{Ni}$ 的分电流效率, $E_{\mathrm{W}} 、 E_{\mathrm{Ni}}$ 为 $\mathrm{W}$ 和 $\mathrm{Ni}$ 的电化当量, 分别为 $0.318 \times 10^{-3} \mathrm{~g} \cdot \mathrm{C}^{-1}$ 和 $0.304 \times 10^{-3} \mathrm{~g} \cdot \mathrm{C}^{-1} ; \Delta \mathrm{G}$ 为镀层重量 $(\mathrm{g}) ; w_{\mathrm{W}} 、 w_{\mathrm{Ni}}$ 是镀层中 $\mathrm{W}$ 和 $\mathrm{Ni}$ 的质量分数; $I 、 t$ 分别为沉积电 流 (A) 和时间 $(\mathrm{s})$.

\section{2 实验结果与讨论}

\section{1 二甲基胺硼烷在 Ni-W 合金电沉积过程中的 作用}

在含有二甲基胺硼烷的 $\mathrm{Ni}-\mathrm{W}$ 合金镀液中，还
原性物质二甲基胺硼烷本身在 Ni-W 合金诱导共沉 积过程中在电极表面上并没有发生阴极还原 ${ }^{[7]}$. 因 此, 可以认为, 其主要作用表现为对镀液中镍的化学 还原。根据文献 [14], 反应过程为

$$
\begin{gathered}
\left(\mathrm{CH}_{3}\right)_{2} \mathrm{NHBH}_{3}+3 \mathrm{Ni}^{2+}+3 \mathrm{H}_{2} \mathrm{O}=3 \mathrm{Ni}+ \\
\left(\mathrm{CH}_{3}\right)_{2} \mathrm{H}_{2} \mathrm{~N}^{+}+\mathrm{H}_{3} \mathrm{BO}_{3}+5 \mathrm{H}^{+} \\
2\left(\mathrm{CH}_{3}\right)_{2} \mathrm{NHBH}_{3}+4 \mathrm{Ni}^{2+}+3 \mathrm{H}_{2} \mathrm{O}=\mathrm{Ni}_{2} \mathrm{~B}+2 \mathrm{Ni}+ \\
2 \mathrm{R}_{2} \mathrm{H}_{2} \mathrm{~N}^{+}+\mathrm{H}_{3} \mathrm{BO}_{3}+1 / 2 \mathrm{H}_{2}+6 \mathrm{H}^{+}
\end{gathered}
$$

反应结果清楚表明: 在 Ni-W 合金电沉积过程中, 镀 液中二甲基胺嗍烷的存在必然导致沉积层 $\mathrm{Ni}$ 含量 提高并含 B, Ni-W-B 合金包含着大量的镍铇和少 量的镍硼化合物。化学沉积 $\mathrm{Ni}$ 过程中产生的 $\mathrm{H}_{3} \mathrm{BO}_{3}$ 在镀液介质中将转变成 $\mathrm{Na}_{2} \mathrm{~B}_{4} \mathrm{O}_{7}$, 特别是在电 沉积过程中剧烈的析氢过程必然导致阴极界面 $\mathrm{pH}$ 值的提高, 从而促进 $\mathrm{Na}_{2} \mathrm{~B}_{4} \mathrm{O}_{7}$ 的生成. 表 1 的实验 结果证明, 所获得的合金电沉积层的 $\mathrm{Ni}$ 含量从镀 液中含 $\mathrm{C}_{2} \mathrm{H}_{10} \mathrm{BN}$ 为零时的 $57.0 \%$ 增大至含 $15 \mathrm{~g}$. $\mathrm{L}^{-1}$ 时的 $65.1 \%, \mathrm{~B}$ 含量约为 $1.2 \%$.

为比较方便, 相对于 Ni-W 合金电沉积, 假设镍 化学还原过程的影响较小, 沉积层中的 $\mathrm{Ni}$ 均来自于 电沉积过程中的贡献. 分别计算 Ni-W 和 Ni-W-B 合金电沉积过程中的电流效率 $\eta$, 以及 $\mathrm{Ni}$ 和 $\mathrm{W}$ 的 分电流效率 $\eta_{\mathrm{Ni}}$ 和 $\eta_{\mathrm{w}}$, 结果如表 1 所示. 显然, $\mathrm{Ni}-\mathrm{W}$ 和 Ni-W-B 合金电沉积电流效率 $\eta$ 不高, 从 $\eta_{\mathrm{W}}$ 值可知, 镀液中存在 $\mathrm{C}_{2} \mathrm{H}_{10} \mathrm{BN}$ 对 $\mathrm{W}$ 的电沉积没 有明显影响, 而 $\eta_{\mathrm{Ni}}$ 的值明显增大, 说明 Ni-W-B 合 金电沉积过程中包含化学沉积镍过程, 结果致使 $\eta_{\mathrm{Ni}}$ 和 $\eta$ 值增大.

图 1 表示在所述的镀液组成和沉积条件下所获 得的 Ni-W-B 合金电沉积层的 X 射线电子能谱 (XPS) 图。图中结果表明, 在结合能为 $187.5 \mathrm{eV}$ 和 $191.7 \mathrm{eV}$ 处出现谱峰, 经对照和推测, 它们分别对 应于镍硼化物 ${ }^{[12,14]}$ 和 $\mathrm{Na}_{2} \mathrm{~B}_{4} \mathrm{O}_{7}$ 物质 ${ }^{[15]}$. 此结果进一 步表明, 在 Ni-W-B 合金电沉积过程中伴随着化学 沉积镍和析氢过程, 而且存在 $\mathrm{Na}_{2} \mathrm{~B}_{4} \mathrm{O}_{7}$ 在沉积层中 的夹杂. 镍嗍化物和夹杂物的存在必然引起以主体

表 1 Ni-W(-B)合金电沉积电流效率和镀层组成

Table 1 Current efficiency and composition of deposited Ni-W and Ni-W-B alloy

\begin{tabular}{ccccccc}
\hline Alloy deposit & $\eta(\%)$ & $\eta_{\mathrm{Ni}}(\%)$ & $\eta_{\mathrm{W}}(\%)$ & $w_{\mathrm{W}}$ & $w_{\mathrm{Ni}}$ & $w_{\mathrm{B}}$ \\
\hline Ni-W & 41.7 & 23.3 & 18.4 & 43.0 & 57.0 & 0 \\
Ni-W-B & 50.2 & 32.5 & 17.7 & 33.7 & 65.1 & 1.2 \\
\hline
\end{tabular}




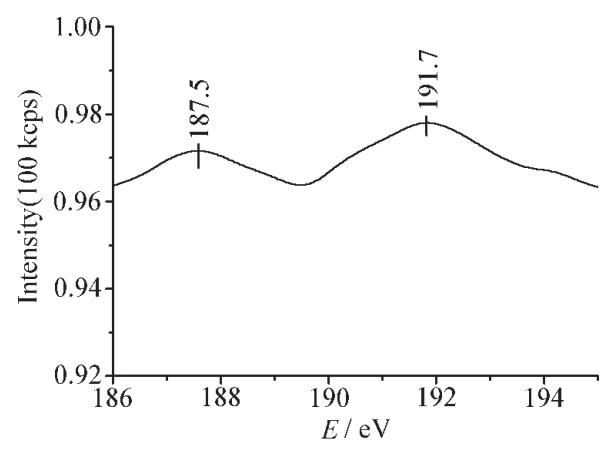

图 1 Ni-W-B 合金镀层的 XPS 谱

Fig. 1 XPS pattern of Ni-W-B alloy electrodeposit

形式存在的 Ni-W 合金固溶体结构的变化，导致晶 格产生进一步的扭曲、缺陷和畸变，从而引起其性 能的变化.

\subsection{Ni-W 和 Ni-W-B 合金电沉积层的热处理}

图 2 为 $\mathrm{Ni}-\mathrm{W}$ 和 Ni-W-B 合金电沉积层的微分 扫描热分析 (DSC) 实验结果, 曲线 1 说明在所述的 热处理温度范围内, Ni-W 合金电沉积层没有明显的 放热峰, 而是在热处理达到 $350{ }^{\circ} \mathrm{C}$ 以上时逐渐有少 量的放热过程; 与 $\mathrm{Ni}-\mathrm{W}$ 合金不同，曲线 2 表明非金 属元素 B 的存在使 Ni-W-B 合金镀层在 $358.9{ }^{\circ} \mathrm{C}$, $377.8{ }^{\circ} \mathrm{C}$ 产生明显的放热峰, 结合 XRD 实验结果, 说 明了该合金的非晶态特征以及在退火中有新相产生.

根据 Ni-W 合金相图 ${ }^{[10],} \mathrm{Ni}-\mathrm{W}$ 合金电沉积层 中 $\mathrm{W}$ 含量超过 $26 \%$ 时将形成过饱和固溶体. 因 此, 在本实验条件下所获得的 $\mathrm{Ni}-43.0 \% \mathrm{~W}$ 镀层由 于其结构上的非稳定性, 在热处理过程中必然转变 成能量较低的稳定态。嗍是 Ni-W-B 合金形成非晶 态结构的主要添加剂 ${ }^{[8]}$, 在热处理过程中, 它的存在

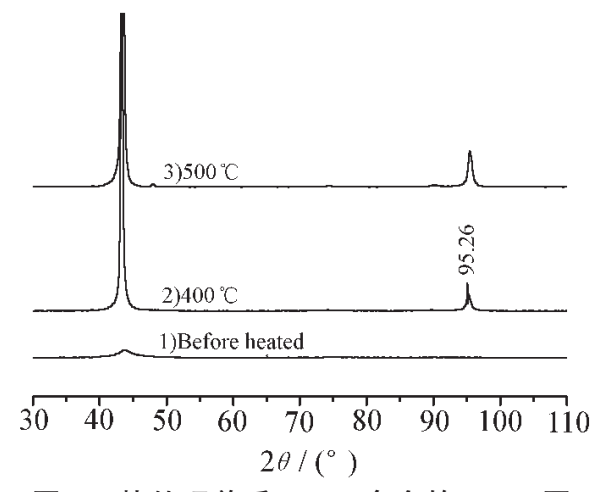

图 3 热处理前后 Ni-W 合金的 XRD 图

Fig. 3 XRD pattern of Ni-W alloy before and after heat treatment

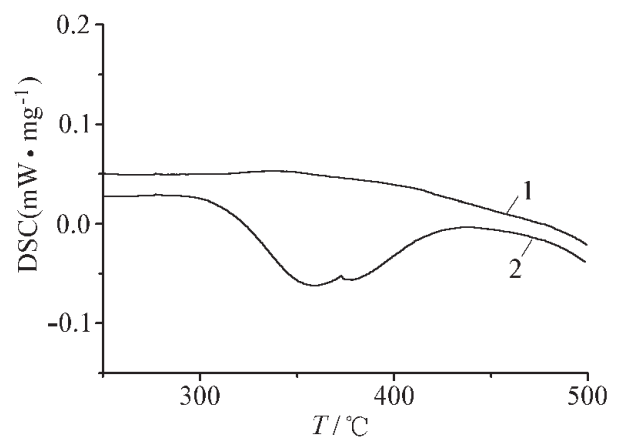

图 2 Ni-W(-B)合金 DSC 图

Fig. 2 DSC plot of Ni-W(-B ) alloy

1) Ni-W alloy; 2)Ni-W-B alloy

也促进了镀层中铇原子的扩散 ${ }^{[10]}$. Ni-W-B 合金包 含着镍铇、镍嗍和 $\mathrm{Na}_{2} \mathrm{~B}_{4} \mathrm{O}_{7}$ 等化合物, 因此, 可以认 为 $\mathrm{DSC}$ 图中 $358.9{ }^{\circ} \mathrm{C}$ 和 $377.8{ }^{\circ} \mathrm{C}$ 的放热峰归因于 新相 $\mathrm{Ni}_{4} \mathrm{~W}$ 的形成和镍硼化合物如 $\mathrm{Ni}_{2} \mathrm{~B} 、 \mathrm{Ni}_{3} \mathrm{~B}$ 等的 产生 .

\section{3 热处理对 Ni-W(-B)合金电沉积层的结构和 显微硬度的影响}

$\mathrm{Ni}-\mathrm{W}$ 合金为置换固溶体。在固溶体中，原子 半径 $(0.137 \mathrm{~nm})$ 较大的 $\mathrm{W}$ 原子周围受到压缩, 原 子半径 $(0.122 \mathrm{~nm})$ 较小的 $\mathrm{Ni}$ 原子周围受到拉伸, 结果必然导致合金晶格产生扭曲、缺陷和胀大, 引 起正畸变, 晶面间距 $d$ 值增大. 图 3 和图 4 分别为 Ni-W 和 Ni-W-B 合金镀层热处理前后的 XRD 图 . 表 2 示出其 XRD 实验结果和显微硬度. 与标准 $\mathrm{Ni}$ 的 $d$ 值 $0.2034 \mathrm{~nm}$ 相比，退火前后 $\mathrm{Ni}-\mathrm{W}$ 合金和 Ni-W-B 合金结构的 $d$ 值均较大.从图 3 可以看 出, 热处理前 $\mathrm{Ni}-\mathrm{W}$ 合金 (曲线 1 ) 的 XRD 图主要出 现对应于 $\mathrm{Ni}$ (111) 晶面的 $2 \theta$ 值为 $43.74^{\circ}$ 的宽化的

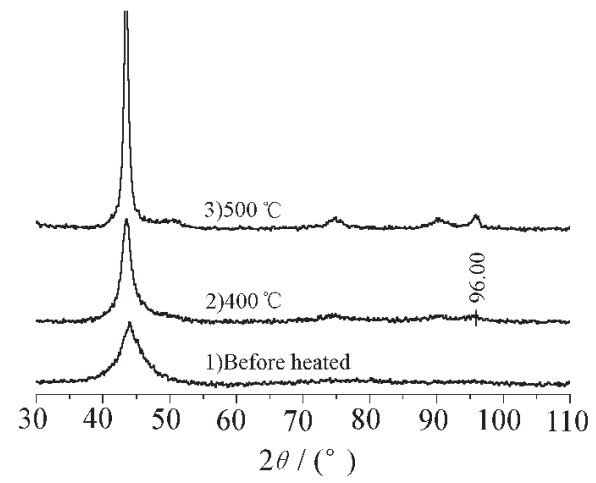

图 4 热处理前后 Ni-W-B 合金的 XRD 图

Fig. 4 XRD pattern of Ni-W-B alloy before and after heat treatment 
表 2 热处理前后镀层的 XRD 实验结果和显微硬度

Table 2 XRD experimental results and microhardness of the deposits before and after heat treatment

\begin{tabular}{|c|c|c|c|c|c|c|}
\hline \multirow[b]{2}{*}{ Alloy deposit } & \multicolumn{3}{|c|}{$\mathrm{Ni}-\mathrm{W}$} & \multicolumn{3}{|c|}{ Ni-W-B } \\
\hline & $\begin{array}{l}\text { Before } \\
\text { heated }\end{array}$ & $\begin{array}{c}\text { heated at } \\
400{ }^{\circ} \mathrm{C}\end{array}$ & $\begin{array}{c}\text { Heated at } \\
500{ }^{\circ} \mathrm{C}\end{array}$ & $\begin{array}{l}\text { Before } \\
\text { heated }\end{array}$ & $\begin{array}{c}\text { Heated at } \\
400{ }^{\circ} \mathrm{C}\end{array}$ & $\begin{array}{c}\text { Heated at } \\
500{ }^{\circ} \mathrm{C}\end{array}$ \\
\hline $2 \theta /\left(^{\circ}\right)$ & 43. 74 & 43.28 & 43.28 & 44.02 & 43.72 & 43.48 \\
\hline$d / \mathrm{nm}$ & 0.2068 & 0. 2089 & 0. 2089 & 0.2055 & 0.2069 & 0.2080 \\
\hline Intensity (CPS) & 609 & $29.3 \times 10^{3}$ & $58.2 \times 10^{3}$ & 454 & 867 & 2186 \\
\hline$D / \mathrm{nm}$ & 3.45 & 12.67 & 17. 15 & 2. 79 & 4. 46 & 8. 53 \\
\hline$H_{v} / \mathrm{kg} \cdot \mathrm{mm}^{-2}$ & 557.2 & 919.8 & 706.8 & 602.9 & 946.2 & 1132.2 \\
\hline
\end{tabular}

衍射峰, 峰强度为 609 , 微晶尺寸为 $3.45 \mathrm{~nm}$, 结合 图 2 结果说明该合金为 $\mathrm{Ni}$ 基置换固溶体的纳米尺 寸合金结构. 分别经过 $400{ }^{\circ} \mathrm{C}$ (曲线 2 ) 和 $500{ }^{\circ} \mathrm{C}$ (曲线 3) 热处理 $120 \mathrm{~min}$ 后, $\mathrm{Ni}-\mathrm{W}$ 合金的晶粒粗化 作用明显, 对应于 $\mathrm{Ni}(111)$ 的衍射峰半峰宽宽化程 度变小, 峰强度由 609 急剧增大到 $29.3 \mathrm{kCPS}$, 并在 95. $26^{\circ}$ 处出现 $\mathrm{Ni}_{4} \mathrm{~W}$ 的特征峰 ${ }^{[10]}$, 微晶尺寸由 3.45 $\mathrm{nm}$ 提高到 $12.67 \mathrm{~nm}$ 和 $17.15 \mathrm{~nm}$, 说明该合金在热 处理过程中发生晶粒粗化过程并析出细小颗粒的金 属化合物 $\mathrm{Ni}_{4} \mathrm{~W}$. 与图 2 曲线 1 结果比较可以推测, 产生的 $\mathrm{Ni}_{4} \mathrm{~W}$ 含量可能很少或者是热处理温度尚不 够高,从而导致该曲线没有出现放热特征峰。

图 4 结合表 2 结果表明, 热处理前 (曲线 1) $\mathrm{Ni}-\mathrm{W}-\mathrm{B}$ 合金的 XRD 图中仅在 $2 \theta$ 值为 $44.02^{\circ}$ 附近 出现半峰宽宽化程度达到 $3.41^{\circ}$ 、峰强度仅为 454 的漫散射峰, 结合图 2 热处理实验结果以及文献 [8, 10] 结果, 说明 Ni-W-B 合金电沉积层呈现显著 的非晶态特征。与 $\mathrm{Ni}-\mathrm{W}$ 合金的研究结果相似，分 别在 $400{ }^{\circ} \mathrm{C}$ (曲线 2 ) 和 $500{ }^{\circ} \mathrm{C}$ (曲线 3 ) 热处理后, $\mathrm{Ni}-\mathrm{W}-\mathrm{B}$ 合金的 $\mathrm{Ni}$ (111) 衍射峰变得尖锐, 峰强度增 大到 867 和 2186 , 并在 $2 \theta$ 值为 $96^{\circ}$ 处出现 $\mathrm{Ni}_{4} \mathrm{~W}$ 的 衍射峰并且强度逐渐增大; 沉积层微晶尺寸则分别 从 $2.79 \mathrm{~nm}$ 提高至 $4.46 \mathrm{~nm}$ 和 $8.53 \mathrm{~nm}$. 尽管 Ni-W-B 合金电沉积层的 X 射线衍射峰强度和微晶 尺寸均随退火温度的提高而增大，但其变化值均比 $\mathrm{Ni}-\mathrm{W}$ 合金电沉积层的小, 可见, 沉积层中 $\mathrm{B}$ 的存在 对合金结构的非晶态过程及其变化有显著的影响.

镀层硬度指镀层对外力所引起的局部表面形变 的抵抗强度. Ni-W 合金电沉积层的显微硬度随镀 层中 $\mathrm{W}$ 含量的提高而增大 ${ }^{[4,10]}$, 与晶块尺寸、相干 应变 (coherency strains)、位错和晶界等 ${ }^{[5]}$ 结构变化 有关. 从 $\mathrm{Ni}-\mathrm{W}$ 合金相图 ${ }^{[10]}$ 可知, 热处理后镀层为
一平衡的相结构, 表现为含 $\mathrm{W} 26 \%$ 的 $\mathrm{Ni}$ 基金属固 溶体和少量金属化合物 $\mathrm{Ni}_{4} \mathrm{~W} .400{ }^{\circ} \mathrm{C}$ 热处理时, 镀 层中分布着细小的 $\mathrm{Ni}_{4} \mathrm{~W}$, 结果导致显微硬度迅速提 高, 达到 $919.8 \mathrm{~kg} \cdot \mathrm{mm}^{-2}$, 如表 2 所示. 而在 500 ${ }^{\circ} \mathrm{C}$ 热处理后, $\mathrm{Ni}-\mathrm{W}$ 合金镀层的晶粒生长, 沉积物粗 化, 并进一步产生晶界, 结果其显微硬度下降至 $706.8 \mathrm{~kg} \cdot \mathrm{mm}^{-2}$, 此实验结果与文献 [10] 的变化结 果一致。

表 2 实验结果进一步表明, Ni-W-B 合金电沉 积层中的 $\mathrm{W}$ 含量较 $\mathrm{Ni}-\mathrm{W}$ 合金中的低, 但热处理前 后该合金电沉积层的显微硬度均较 Ni-W 合金的 大，可见 Ni-W 合金电沉积层中 $\mathrm{B}$ 的引人导致其显 微硬度有明显的增大。Ni-W-B 合金电沉积层热处 理后产生细小颗粒的金属化合物 $\mathrm{Ni}_{4} \mathrm{~W}, \mathrm{Ni}_{2} \mathrm{~B}$ 和 $\mathrm{Ni}_{3} \mathrm{~B}^{[10,12,14]}$ 等沉积物, 导致其显微硬度迅速提高. 镀层中 $\mathrm{B}$ 的存在可能使其晶粒生长速度变慢 ${ }^{[10]}$. 由 于晶粒生长缓慢, Ni-W-B 合金在热处理条件下的 显微硬度变化趋势不同于 $\mathrm{Ni}-\mathrm{W}$ 合金，在 $500{ }^{\circ} \mathrm{C}$ 处 理 $2 \mathrm{~h}$ 后显微硬度仍继续从 $400{ }^{\circ} \mathrm{C}$ 时的 $946.2 \mathrm{~kg}$. $\mathrm{mm}^{-2}$ 提高至 $1132.2 \mathrm{~kg} \cdot \mathrm{mm}^{-2}$, 与铬镀层的显微硬 度相近。

\section{3 结 论}

在 Ni-W-B 合金电沉积过程中伴随着析氢和化 学沉积镍过程以及 $\mathrm{Na}_{2} \mathrm{~B}_{4} \mathrm{O}_{7}$ 在镀层中的夹杂; Ni-W 合金电沉积层为纳米晶结构，而 Ni-W-B 合金为非 晶态结构, 合金热处理过程中产生 $\mathrm{Ni}_{4} \mathrm{~W}$, 镍硼化合 物等细小沉积物。镀层中分散着的沉淀物导致镀层 显微硬度提高，在 $400{ }^{\circ} \mathrm{C}$ 下 $\mathrm{Ni}-\mathrm{W}$ 合金有最大的硬 度达 $919.8 \mathrm{~kg} \cdot \mathrm{mm}^{-2}$, 而在 $500{ }^{\circ} \mathrm{C}$ 下 $\mathrm{Ni}-\mathrm{W}-\mathrm{B}$ 合金 有最大的硬度达 $1132.2 \mathrm{~kg} \cdot \mathrm{mm}^{-2}$. 


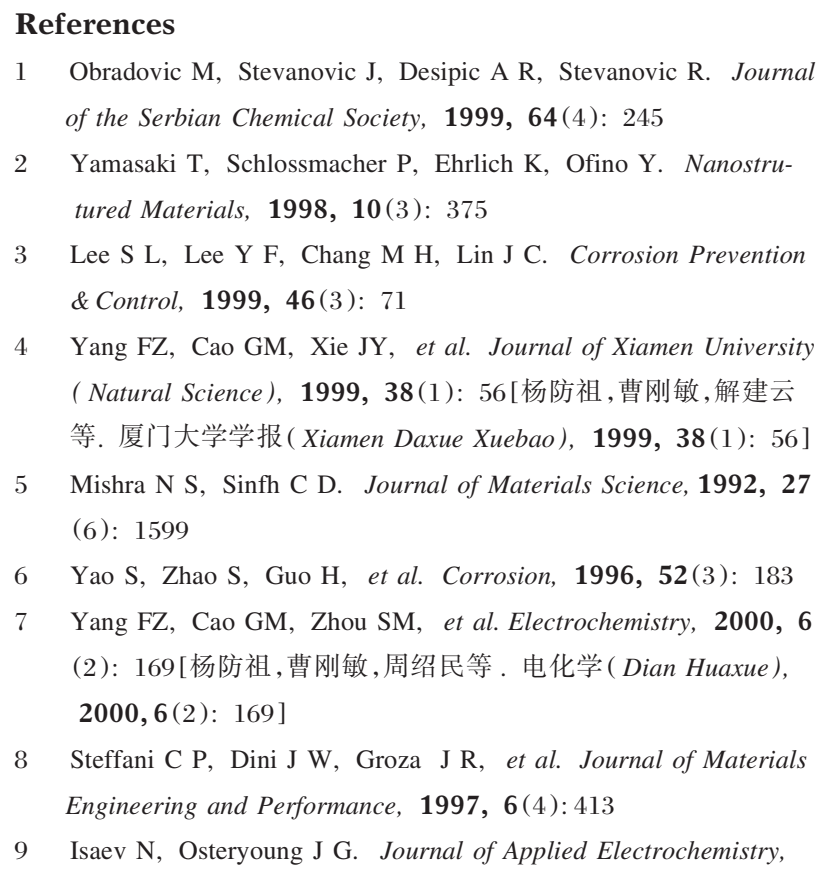

1995, 25(12): 1091

10 Gretchen Graef, Ken Anderson, Joanna Groza, Ahmet Palazoflu. Materials and Engineering B, 1996, 41(2): 253

11 Croopnick G A, Scruggs D M. Metal Finishing, 1994, 92(4): 13

12 Morishita M, Koyama K, Maeda K, Zhang G F. Materials Transactions JIM, 1999, 40(7): 600

13 Chen GZ, Huang XZ, Liu WY, et al. Ultraviolet-visible Spectrometry (Second volume). Beijing: Atomic Energy Press House, 1987, 277p [陈国珍, 黄贤智, 刘文远 等编著. 紫外可 见分光光度法 (下册), 北京: 原子能出版社, 1987. 277]

14. Wolfgang Riedel(Write), Luo SF(Translate), Gu MY (Correct). Electroless Nickel Plating. Shanghai: Shanghai Jiaotong University Press House, 1996. 27p[沃尔夫冈・里德尔著, 罗守福 译, 顾明元校. 化学镀镍, 上海: 上海交通大学出版社, 1996. 27]

15 Briggs D, Seah M P. Practical Surface Analysis (Second Edition), Volume 1: Auger and X-ray Photoelectron Spectroscopy. John Wiley \& Sons Ltd, 1990. 599

\title{
Structure and Property of Ni-W-B Alloy Electrodeposits Before and After Heat Treatment*
}

\author{
Cao Gang-Min Yang Fang-Zu Huang Ling Niu Zhen-Jiang Xu Shu-Kai Zhou Shao-Min \\ ( Chemistry Department, State Key Laboratory for Physical Chemistry of Solid Surfaces, Institute of Physical Chemistry, \\ Xiamen University, Xiamen 361005 )
}

\begin{abstract}
The structure and microhardness of Ni-W-B alloy were studied by means of electrochemical techniques, XPS, DSC and XRD before and after heat treatment. The results showed that the process of Ni-W-B alloy electrodeposition was accompanied by the chemical deposition of $\mathrm{Ni}$ and adulteration of $\mathrm{Na}_{2} \mathrm{~B}_{4} \mathrm{O}_{7}$ in the deposit. Ni-W alloy presented in nanocrystalline structure and Ni-W-B in amorphous structure were both coarsened in grain size and led to the evolution of $\mathrm{Ni}_{4} \mathrm{~W}$, and precipitation of $\mathrm{Ni}-\mathrm{B}$ compound such as $\mathrm{Ni}_{2} \mathrm{~B}$, $\mathrm{Ni}_{3} \mathrm{~B}$ in $\mathrm{Ni}-\mathrm{W}-\mathrm{B}$ alloy after heat treatment. The maximum microhardness could be observed for Ni-W alloy at the value of $919.8 \mathrm{~kg} \cdot \mathrm{mm}^{-2}$ after heat treatment for 2 hours at temperature of $400{ }^{\circ} \mathrm{C}$ and Ni-W-B alloy at $1132.2 \mathrm{~kg} \cdot$ $\mathrm{mm}^{-2}\left(500{ }^{\circ} \mathrm{C}, 2 \mathrm{hrs}\right)$.
\end{abstract}

Keywords: Ni-W-B alloy, Electrodeposition, Structure, Microhardness, Heat treatment 